\title{
Predictors of decreased left ventricular function subsequent to follow-up echocardiography after percutaneous coronary intervention following acute ST-elevation myocardial infarction
}

\author{
DONG-HEE KIM, CHANG-BUM PARK，EUN-SUN JIN，HUI-JEONG HWANG， \\ IL SUK SOHN, JIN-MAN CHO and CHONG-JIN KIM \\ Department of Cardiology, Kyung Hee University Hospital at Gangdong, \\ Kyung Hee University, Seoul 05278, Republic of Korea \\ Received March 6, 2016; Accepted February 24, 2017
}

DOI: $10.3892 /$ etm.2018.5962

\begin{abstract}
The preferred treatment for patients with ST elevation myocardial infarction (STEMI) is primary percutaneous coronary intervention (PCI). However, not all patients improve or maintain heart function following primary PCI, and certain patients may experience decreased heart function. The present study investigated factors associated with left ventricular (LV) dysfunction, and improvement or deterioration of LV ejection fraction (LVEF) at follow-up echocardiography following successful primary PCI. The clinical outcomes following primary PCI were also investigated. The present study assessed 4,044 patients who underwent primary PCI following a diagnosis of STEMI between January 2008 and March 2012. A total of 1,736 patients who underwent echocardiography between 30 days and 1 year after STEMI and PCI, and who had completed clinical follow-up, were included in the present study. A total of 243 patients (14.0\%) demonstrated LV dysfunction at follow-up echocardiography. Multivariate analysis revealed that LV dysfunction $(\leq 40 \%)$ at index STEMI, LVEF at index admission, renal insufficiency (creatinine $\geq 1.4 \mathrm{mg} / \mathrm{dl}$ ), peak creatine kinase (CK) and peak CK $\mathrm{MB}$ isoenzyme (CKMB) were independent predictors of $\mathrm{LV}$ dysfunction at follow-up. Independent predictors for the deterioration of LVEF at follow-up were dyslipidemia, LVEF at index admission, $\mathrm{LVEF} \leq 40 \%$ at index admission, peak CK and peak troponin-I. Furthermore, being male, having no history of coronary artery disease, pre-thrombolysis in myocardial infarction (TIMI) flow, LVEF at index admission, LVEF $\leq 40 \%$ at index admission, peak CKMB and peak troponin I were independent predictors of LVEF improvement at follow-up. One-year major adverse cardiac events were significantly increased in the LV
\end{abstract}

Correspondence to: Professor Chang-Bum Park, Department of Cardiology, Kyung Hee University Hospital at Gangdong, Kyung Hee University, 892 Dongnam-ro, Gangdong-gu, Seoul 05278, Republic of Korea

E-mail:wwwpcb@hanmail.net

Key words: myocardial infarction, coronary occlusion, angioplasty dysfunction group compared with patients who did not exhibit LV dysfunction according to Cox regression analysis (13.6 vs. $20.4 \%$; $\mathrm{P}=0.017$ ). Therefore, the present study may provide valuable prognostic information for clinicians to advise patients who experience LV dysfunction despite having undergone successful primary PCI. Additional management is required in patients with these high-risk features following STEMI.

\section{Introduction}

ST-elevation acute myocardial infarction (STEMI) is a clinical syndrome defined by characteristic symptoms of myocardial ischemia in association with persistent electrocardiographic ST elevation and subsequent release of biomarkers of myocardial necrosis (1). Worldwide, ischemic heart disease in the single most common cause of fatality and its frequency has increased. Approximately 683,000 patients were discharged from US hospitals with the diagnosis of acute coronary syndrome (2). Notably, STEMI comprised 25-40\% of myocardial infarction presentations in 2009 (2). Recently, early reperfusion by mechanical or pharmacological means and adjunctive antithrombotic treatment has been proven to lower mortality $(3,4)$. Therefore, several generations of international and national guidelines have been presented to support that the standard treatment for patients with STEMI is primary percutaneous coronary intervention (PCI) $(1,2,5)$. However, STEMI remains a major health issue worldwide despite the improvement of efficacy and safety of several novel treatments available for patients with STEMI (6). Recent studies have highlighted a fall in acute and long-term mortality following STEMI in parallel with greater use of reperfusion therapy, including PCI $(7,8)$. Notably, primary PCI in patients with STEMI can limit the infarct size and preserves left ventricular (LV) systolic function $(6,9)$. However, a problem with this approach is that not all patients with STEMI improve or maintain heart function following PCI. In fact, 4.7-8.6\% patients may experience decreased heart function even after undergoing successful primary PCI $(10,11)$. As congestive heart failure (CHF) after PCI in STEMI is known as a major cause of morbidity and mortality, which may lead to hospitalization and consumption of heart-care resources (12), identification of 
patients with high risk of CHF may aid in the selection of more appropriate post-infarction therapies such as optimal doses of angiotensin-converting enzyme inhibitors, beta blockers or aldosterone antagonists (13-15).

The present study investigated predictors associated with LV dysfunction and the improvement or deterioration of LV ejection fraction (LVEF) at follow-up echocardiography within 1 year of STEMI. The prognosis of patients with LV dysfunction who underwent successful primary PCI following STEMI was also investigated.

\section{Patients and methods}

Study population. All 4,044 patients received primary PCI following a diagnosis of STEMI from the Korea Working Group on Myocardial Infarction (KorMI) registry (www. kamir.or.kr) between January 2008 and March 2012 were included in the present study. The number of male and female patients was 2,613 (64.6\%) and 1,431 (35.4\%), respectively. The mean age of the enrolled patients was $62.3 \pm 12.7$. KorMI is a multicenter, nationwide, web-based database supported by the Korean Society of Cardiology. A total of 52 centers in Korea attended this registry and gathered patients' information. Written informed consent was obtained from all patients enrolled in the current study. The present study was approved by the Institutional Review Board at Kyung Hee University Hospital at Gangdong (IRB Approval no. KHNMC 2010-01-065).

The diagnosis of STEMI was based on a suggestive history, with ST elevation $>2 \mathrm{~mm}$ in more than two precordial leads, ST elevation $>1 \mathrm{~mm}$ in two limb leads or new left bundle branch block on the 12-lead electrocardiogram with a concomitant increase of cardiac markers more than two times the upper limit of normal levels (troponin I, 0-0.1 ng/ml; creatinine kinase-MB, 0-0.3 ng/ml).

LV dysfunction was defined as $\leq 40 \%$ of LVEF. Follow-up LVEF was evaluated using two-dimensional echocardiography, performed within 1 year after the index PCI. Improvement of LV function was defined as $>5 \%$ increase of LVEF at follow-up echocardiography. Deterioration of LV function was defined as $<5 \%$ decrease of LVEF at follow-up echocardiography. One-year major adverse cardiac events (MACEs) included cardiac death, noncardiac death, myocardial infarction, target vessel revascularization and non-target vessel revascularization that occurred within 1 year after the index procedure.

Statistical analysis. Continuous variables with normal distributions were expressed as the mean \pm standard deviation, and categorical variables were compared using $\chi^{2}$ or Fisher's exact tests if the expected value of the variables was $\leq 5$ in at least one group. Univariate regression analysis was performed on variables with $\mathrm{P}$-values $<0.05$ to identify determinants of $\mathrm{LV}$ dysfunction, and deterioration and improvement of LVEF at follow-up. Variables found to be significant by univariate analysis also underwent multivariate analysis to determine their independent relationship to restenosis. Cox regression analysis was conducted to compare survival between the LV dysfunction and non-dysfunction groups with independent predictors identified by multivariate analysis. Statistical analyses were performed using SPSS v. 12.5 for Windows (SPSS,
Inc., Chicago, IL, USA) and $\mathrm{P}<0.05$ was considered to indicate a statistically significant difference.

\section{Results}

Baseline characteristics. Out of the 4,044 patients who underwent primary PCI following diagnosis of STEMI, 1,736 patients were enrolled who received initial and follow-up echocardiography between 3 months and 1 year after index PCI and who had completed clinical follow-up. Of these patients, $243(14.0 \%)$ exhibited LV dysfunction, with LVEF $\leq 40 \%$ at follow-up echocardiography.

Predictors for follow-up LV dysfunction. Demographic, clinical and angiographic characteristics are presented in Tables I and II. Univariate analysis demonstrated that significant predictors of LV dysfunction included age $(\mathrm{P}<0.001)$, an age $>70$ years $(\mathrm{P}<0.001)$, higher $\mathrm{BMI}$ $(\mathrm{P}=0.008)$, history of cerebrovascular disease $(\mathrm{P}=0.006)$, prior ischemic heart disease $(\mathrm{P}=0.016)$, Killip class $(6,7) \geq 2$ $(\mathrm{P}<0.01), \mathrm{LVEF} \leq 40 \%$ at index admission $(\mathrm{P}<0.001)$, renal insufficiency (creatinine $\geq 1.4 \mathrm{mg} / \mathrm{dl}$; $\mathrm{P}<0.001$ ), higher peak creatine kinase $(\mathrm{CK})$ level $(\mathrm{P}<0.001)$, higher peak $\mathrm{CK} \mathrm{MB}$ isoenzyme $(\mathrm{CKMB})$ level $(\mathrm{P}<0.001)$, higher peak troponin I level $(\mathrm{P}<0.001), \mathrm{B}$-natriuretic peptide level $(\mathrm{P}=0.001)$, use of a bare metal stent, 1 vessel disease $(\mathrm{P}<0.001)$, triple vessel disease $(\mathrm{P}<0.001), \mathrm{PCI}$ at left anterior descending (LAD) artery $(\mathrm{P}<0.001), \mathrm{PCI}$ at right coronary artery $(\mathrm{P}<0.001)$, stent number at culprit lesion $(\mathrm{P}=0.015)$ and a pre-procedural thrombolysis in myocardial infarction (TIMI) grade $<1$ ( $\mathrm{P}=0.016$; Tables I and II). Multivariate logistic regression analysis was conducted using the significant univariate predictors to determine their association with lack of LVEF recovery (Table III). In multivariate analysis, LV dysfunction $(\mathrm{LVEF} \leq 40 \%)$ at index STEMI $[\mathrm{P}=0.023$; odds ratio $(\mathrm{OR})$, 3.062; 95\% confidence interval (CI), 1.124-5.000], LVEF at index admission ( $\mathrm{P}<0.001$; OR, 1.080; 95\% CI, 0.892-0.962), renal insufficiency (creatinine $\geq 1.4 \mathrm{mg} / \mathrm{dl} ; \mathrm{P}=0.039$; OR, 2.637; 95\% CI, 1.038-4.237), peak CK ( $\mathrm{P}=0.006$; OR, 1.000; 95\% CI, 1.000-1.000), peak CKMB ( $\mathrm{P}=0.034$; OR, 0.999; 95\% CI, 0.998-1.000) were identified to be independent predictors of LV dysfunction at follow-up.

Predictors for deterioration and improvement of LVEF at follow-up echocardiography. The number of patients that exhibited deterioration and improvement of LVEF at follow-up echocardiography was 293 and 658, respectively. Their demographic, clinical and angiographic characteristics are listed in Tables IV and V. The patients that demonstrated improvement of LVEF exhibited a significantly reduced incidence of dyslipidemia $(\mathrm{P}=0.004)$, reduced peak $\mathrm{CK}$ level $(\mathrm{P}<0.001)$, reduced peak $C K M B$ level $(\mathrm{P}=0.002)$ and reduced peak troponin I level $(\mathrm{P}<0.001)$ compared with the group that experienced deterioration of LVEF. One-year MACEs were significantly higher in the group that underwent deterioration of LVEF at follow-up compared with the group that underwent improvement of $\operatorname{LVEF}(\mathrm{P}=0.036)$.

In multivariate analysis, independent predictors for the deterioration of LVEF at follow-up echocardiography were dyslipidemia ( $\mathrm{P}=0.042$; OR, 1.697; 95\% CI, 1.020-2.823), 
Table I. Baseline clinical characteristics of patients with left ventricular dysfunction.

\begin{tabular}{|c|c|c|c|}
\hline \multirow[b]{2}{*}{ Characteristic } & \multicolumn{2}{|c|}{ Left ventricular dysfunction } & \multirow[b]{2}{*}{ P-value } \\
\hline & No $(n=1493)$ & Yes $(n=243)$ & \\
\hline Age, years & $60.2 \pm 11.9$ & $63.6 \pm 12$ & $<0.001^{\mathrm{a}}$ \\
\hline Age $\geq 70$ years, $\%$ & 25.6 & 38.7 & $<0.001^{\mathrm{a}}$ \\
\hline Male, $\%$ & 76.1 & 74.8 & 0.686 \\
\hline BMI & $24.2 \pm 3.1$ & $23.6 \pm 3.2$ & $0.008^{\mathrm{a}}$ \\
\hline Hypertension, \% & 42.6 & 46.0 & 0.329 \\
\hline Diabetes, $\%$ & 23.1 & 28.8 & 0.062 \\
\hline Smoking, \% & 63.7 & 62.2 & 0.665 \\
\hline Dyslipidemia, $\%$ & 7.6 & 9.0 & 0.440 \\
\hline History of cerebrovascular diseases, $\%$ & 3.3 & 7.4 & $0.006^{\mathrm{a}}$ \\
\hline Peripheral artery disease, $\%$ & 0.5 & 1.6 & 0.074 \\
\hline Symptom to balloon time, $\mathrm{hr}$ & $12.5 \pm 19.6$ & $11.5 \pm 17.3$ & 0.437 \\
\hline Prior ischemic heart disease, $\%$ & 8.8 & 12.3 & $0.016^{\mathrm{a}}$ \\
\hline Family history of ischemic heart disease, $\%$ & 7.5 & 6.5 & 0.692 \\
\hline Prior heart failure, $\%$ & 1.3 & 0.1 & 0.022 \\
\hline Killip class $\geq 2, \%$ & 6.7 & 13.9 & $<0.001^{\mathrm{a}}$ \\
\hline LVEF $\leq 40 \%$ at index admission, $\%$ & 8.3 & 55.6 & $<0.001^{\mathrm{a}}$ \\
\hline LVEF, \% at index admission & $52.7 \pm 10.7$ & $39.0 \pm 8.9$ & $<0.001^{\mathrm{a}}$ \\
\hline Renal insufficiency (Creatinine $\geq 1.4 \mathrm{mg} / \mathrm{dl}$ ), $\%$ & 10.8 & 17.4 & $<0.001^{\mathrm{a}}$ \\
\hline Creatinine, $\mathrm{mg} / \mathrm{dl}$ & $1.1 \pm 0.9$ & $1.2 \pm 1.3$ & $0.027^{\mathrm{a}}$ \\
\hline hs-CRP, mg/dl & $14.7 \pm 67.6$ & $13.2 \pm 42.8$ & 0.767 \\
\hline Total cholesterol, mg/dl & $187.3 \pm 43.2$ & $181.5 \pm 42.0$ & 0.067 \\
\hline Peak CK, ng/dl & $1,812.2 \pm 2,201.1$ & $2,948.0 \pm 2,883.3$ & $<0.001^{\mathrm{a}}$ \\
\hline Peak CKMB, ng/dl & $198.5 \pm 301.5$ & $359.2 \pm 712.3$ & $<0.001^{\mathrm{a}}$ \\
\hline Peak Troponin I, ng/dl & $52.5 \pm 70.6$ & $98.1 \pm 112.6$ & $<0.001^{\mathrm{a}}$ \\
\hline $\mathrm{BNP}, \mathrm{pg} / \mathrm{dl}$ & $441.2 \pm 1,862.1$ & $2,813.0 \pm 10,545.7$ & $0.001^{\mathrm{a}}$ \\
\hline Drug-eluting stent, $\%$ & 93.9 & 90.1 & 0.091 \\
\hline MACE, $\%$ & 13.6 & 20.4 & $0.008^{\mathrm{a}}$ \\
\hline
\end{tabular}

LVEF at index admission $(\mathrm{P}<0.001 ; \mathrm{OR}, 1.122 ; 95 \% \mathrm{CI}$, 1.101-1.144), LVEF $\leq 40 \%$ at index admission $(\mathrm{P}<0.001$; OR, 4.234; 95\% CI, 2.150-8.340), peak CK ( $\mathrm{P}<0.001$; OR, $1.000 ; 95 \% \mathrm{CI}, 1.000-1.000)$ and peak troponin I level $(\mathrm{P}<0.001$, OR: 1.004, 95\% CI: 1.002-1.006). Whereas, being male $(\mathrm{P}=0.041$; OR, 1.330; 95\% CI, 1.012-1.747), a history of coronary artery disease $(\mathrm{P}=0.001$; OR, 0.412; 95\% CI, 0.250-0.679), pre-TIMI flow ( $\mathrm{P}=0.028$; OR, 1.082; 95\% CI, 1.065-1.099), LVEF at index admission $(\mathrm{P}<0.001$; OR, 1.082; 95\% CI, 1.065-1.099), LVEF $\leq 40 \%$ at index admission ( $\mathrm{P}=0.006$; OR, 1.851; 95\% CI, 1.189-2.881), peak CKMB (P=0.001, OR: 1.001, 95\% CI: 1.000-1.002), and peak troponin I $(\mathrm{P}<0.001 ; \mathrm{OR}, 1.003 ; 95 \% \mathrm{CI}, 1.001-1.004)$ were independent predictors of the improvement of LVEF at follow-up echocardiography.

Clinical outcomes of follow-up LV dysfunction. The cumulative survival among patients with LV dysfunction compared to those without LV dysfunction using Cox regression analysis (Fig. 1). One-year MACEs were significantly increased in patients with follow-up LV dysfunction (LV dysfunction vs. no dysfunction, 20.4 vs. $13.6 \%$; $=0.017)$.

\section{Discussion}

The present study demonstrated that LV dysfunction (LVEF $\leq 40 \%$ ) at index STEMI, LVEF renal insufficiency (creatinine $\geq 1.4 \mathrm{mg} / \mathrm{dl}$ ), and high peak $\mathrm{CK}$ and peak CKMB are associated with LV dysfunction at follow-up echocardiography. Furthermore, it was determined that LV dysfunction at follow-up echocardiography is associated with a higher risk of MACEs at 1 year. Although the development of heart failure following STEMI is a recognized predictor of poor outcomes (16-18), there are limited data on the incidence and determinants of heart failure following STEMI treated with primary PCI with drug-eluting stent. Furthermore, the 
Table II. Baseline angiographic characteristics in lesion by left ventricular dysfunction.

Left ventricular dysfunction

\begin{tabular}{|c|c|c|c|}
\hline \multirow[b]{2}{*}{ Characteristic } & & \multirow[b]{2}{*}{ P-value } \\
\hline & No $(n=1493)$ & Yes $(n=243)$ & \\
\hline \multicolumn{4}{|l|}{ Involved vessel, \% } \\
\hline 1-vessel disease & 50.6 & 38.2 & $<0.001^{\mathrm{a}}$ \\
\hline 2-vessel disease & 29.0 & 30.0 & 0.170 \\
\hline 3-vessel disease & 18.2 & 28.3 & $<0.001^{\mathrm{a}}$ \\
\hline Left main disease & 1.6 & 3.2 & 0.122 \\
\hline \multicolumn{4}{|l|}{ Lesion target vessel, $\%$} \\
\hline Left anterior descending & 47.7 & 74.6 & $<0.001^{\mathrm{a}}$ \\
\hline Right coronary & 39.9 & 17.4 & $<0.001^{\mathrm{a}}$ \\
\hline Left circumflex & 9.6 & 6.2 & 0.093 \\
\hline Left main & 1.5 & 1.6 & 0.782 \\
\hline ACC/AHA lesion class B $2 / \mathrm{C}, \%$ & 77.0 & 81.4 & 0.435 \\
\hline Pre-TIMI flow grade $0-1, \%$ & 68.1 & 75.8 & $0.016^{\mathrm{a}}$ \\
\hline Post-TIMI flow grade $0-1, \%$ & 1.3 & 2.4 & 0.250 \\
\hline Total stent number & $1.3 \pm 0.6$ & $1.4 \pm 0.6$ & 0.384 \\
\hline Stent number at culprit lesion & $1.0 \pm 0.1$ & $1.0 \pm 0.1$ & $0.015^{\mathrm{a}}$ \\
\hline Stent length, mm & $25.1 \pm 6.0$ & $25.6 \pm 6.9$ & 0.587 \\
\hline Stent diameter, mm & $3.2 \pm 0.4$ & $3.1 \pm 0.3$ & 0.301 \\
\hline
\end{tabular}

Data are presented as the mean \pm standard deviation unless otherwise specified. ${ }^{a} \mathrm{P}<0.05$. TIMI, thrombolysis in myocardial infarction. ACC/AHA, American College of Cardiology/American Heart Association; TIMI, thrombolysis in myocardial infarction.

Table III. Univariate and multivariate logistic regression analysis associated with left ventricular dysfunction at follow-up echocardiography.

\begin{tabular}{|c|c|c|c|c|c|}
\hline Characteristic & $\begin{array}{c}\text { Univariate } \\
\text { analysis P-value }\end{array}$ & $95 \% \mathrm{CI}$ & $\begin{array}{c}\text { Multivariate } \\
\text { analysis P-value }\end{array}$ & OR & $95 \% \mathrm{CI}$ \\
\hline Age $>70$ years & $<0.001^{\mathrm{a}}$ & $1.351-2.380$ & NS & - & - \\
\hline Age & $<0.001^{\mathrm{a}}$ & $1.012-1.036$ & NS & - & - \\
\hline BMI & $0.008^{\mathrm{a}}$ & $1.016-1.113$ & NS & - & - \\
\hline Cerebral vascular accident & $0.003^{\mathrm{a}}$ & $1.322-4.032$ & NS & - & - \\
\hline Prior ischemic heart disease & $<0.001^{\mathrm{a}}$ & $1.494-3.424$ & NS & - & - \\
\hline Prior heart failure & $0.015^{\mathrm{a}}$ & $1.547-55.555$ & NS & - & - \\
\hline Killip class $\geq 2$ & $<0.001^{\mathrm{a}}$ & $1.631-2.941$ & NS & - & - \\
\hline LV dysfunction ( $\mathrm{EF} \leq 40 \%)$ at index admission & $<0.001^{\mathrm{a}}$ & $10.000-19.323$ & $0.023^{\mathrm{a}}$ & 3.062 & $1.124-5.000$ \\
\hline LVEF \% at index admission & $<0.001^{\mathrm{a}}$ & $0.862-0.892$ & $<0.001^{\mathrm{a}}$ & 1.080 & $0.892-0.962$ \\
\hline Renal insufficiency (Creatinine $\geq 1.4$ mg/dl) & $0.004^{\mathrm{a}}$ & $1.197-2.512$ & $0.039^{\mathrm{a}}$ & 2.637 & $1.038-4.237$ \\
\hline Creatinine & $0.040^{\mathrm{a}}$ & $1.005-1.231$ & NS & - & - \\
\hline Peak CK & $<0.001^{\mathrm{a}}$ & $1.000-1.000$ & $0.006^{\mathrm{a}}$ & 1.000 & $1.000-1.000$ \\
\hline Peak CKMB & $<0.001^{\mathrm{a}}$ & $0.999-1.000$ & $0.034^{\mathrm{a}}$ & 0.999 & $0.998-1.000$ \\
\hline Peak troponin I & $<0.001^{\mathrm{a}}$ & 0.993-0.996 & NS & - & - \\
\hline BNP & $0.029^{\mathrm{a}}$ & $1.000-1.000$ & NS & - & - \\
\hline 3-vessel disease & $<0.001^{\mathrm{a}}$ & $1.308-2.427$ & NS & - & - \\
\hline Culprit lesion at left anterior descending artery & $<0.001^{\mathrm{a}}$ & $2.272-4.201$ & NS & - & - \\
\hline Pre-TIMI flow grade $0-1$ & $0.017^{\mathrm{a}}$ & $1.072-2.013$ & NS & - & - \\
\hline
\end{tabular}

${ }^{\mathrm{a}} \mathrm{P}<0.05$. CI, confidence interval; OR, odds ratio; LVEF, left ventricular ejection fraction; BMI, body mass index; CRP, C-reactive protein; CK, creatine kinase; CKMB, creatine kinase MB isoenzyme; BNP, B-natriuretic peptide; TIMI, thrombolysis in myocardial infarction; NS, not statistically significant. 
Table IV. Baseline clinical characteristics of patients demonstrating deterioration or improvement of left ventricular function at follow-up echocardiography.

Left ventricular dysfunction

\begin{tabular}{|c|c|c|c|}
\hline \multirow[b]{2}{*}{ Characteristic } & & & \multirow[b]{2}{*}{ P-value } \\
\hline & Deterioration $(\mathrm{n}=293)$ & Improvement $(\mathrm{n}=658)$ & \\
\hline Age, years & $60.8 \pm 11.8$ & $60.5 \pm 11.9$ & 0.776 \\
\hline Age $\geq 70$ years, $\%$ & 26.9 & 26.1 & 0.755 \\
\hline Male, $\%$ & 75.7 & 72.9 & 0.361 \\
\hline BMI & $24.1 \pm 3.3$ & $24.2 \pm 3.1$ & 0.604 \\
\hline Hypertension, \% & 41.9 & 43.7 & 0.581 \\
\hline Diabetes, $\%$ & 22.1 & 24.3 & 0.460 \\
\hline Smoking, \% & 63.1 & 60.6 & 0.546 \\
\hline Dyslipidemia, \% & 11.9 & 6.3 & $0.004^{\mathrm{a}}$ \\
\hline Cerebral vascular accident, $\%$ & 5.8 & 3.1 & 0.058 \\
\hline Peripheral artery disease, $\%$ & 0.0 & 0.6 & 0.318 \\
\hline Prior ischemic heart disease, $\%$ & 8.5 & 5.7 & 0.170 \\
\hline Family history of ischemic heart disease, $\%$ & 9.8 & 7.1 & 0.154 \\
\hline Prior heart failure, $\%$ & 0.3 & 0.3 & 1.000 \\
\hline Killip class $\geq 2, \%$ & 22.5 & 24.1 & 0.507 \\
\hline LVEF $\leq 40 \%$ at index admission, $\%$ & 8.8 & 20.6 & $<0.001^{\mathrm{a}}$ \\
\hline LVEF \% at index admission & $57.4 \pm 12.3$ & $47.0 \pm 10.5$ & $<0.001^{\mathrm{a}}$ \\
\hline LVEF \% at follow-up & $46.3 \pm 13.1$ & $59.7 \pm 10.0$ & $<0.001^{\mathrm{a}}$ \\
\hline Renal insufficiency (Creatinine $\geq 1.4 \mathrm{mg} / \mathrm{dl}), \%$ & 13.9 & 12.0 & 0.387 \\
\hline Creatinine, $\mathrm{mg} / \mathrm{dl}$ & $1.1 \pm 0.9$ & $1.1 \pm 0.9$ & 0.514 \\
\hline hs-CRP, mg/dl & $12.0 \pm 56.8$ & $19.2 \pm 77.7$ & 0.187 \\
\hline Total cholesterol, mg/dl & $185.8 \pm 44.2$ & $186.8 \pm 44.4$ & 0.766 \\
\hline Peak CK, ng/dl & $2,518.1 \pm 2,670.8$ & $1,864.2 \pm 2,534.2$ & $<0.001^{\mathrm{a}}$ \\
\hline Peak CKMB, ng/dl & $300.3 \pm 641.9$ & $198.0 \pm 357.0$ & $0.002^{\mathrm{a}}$ \\
\hline Peak troponin I, ng/dl & $82.1 \pm 99.8$ & $51.2 \pm 65.8$ & $<0.001^{\mathrm{a}}$ \\
\hline $\mathrm{BNP}, \mathrm{pg} / \mathrm{ml}$ & $794.1 \pm 4,562.2$ & $1,504.9 \pm 7,705.8$ & 0.515 \\
\hline Drug-eluting stent, $\%$ & 100.0 & 100.0 & 1.000 \\
\hline MACE, \% & 18.0 & 12.9 & $0.036^{\mathrm{a}}$ \\
\hline
\end{tabular}

Data are presented as the mean \pm standard deviation unless otherwise specified. ${ }^{a} \mathrm{P}<0.05$. BMI, body mass index; LVEF, left ventricular ejection fraction; hs-CRP, high-sensitivity C-reactive protein; CK, creatine kinase; CKMB, creatine kinase MB isoenzyme; MACE, major adverse cardiac event; BNP, B-natriuretic peptide.

definition of heart failure has varied in different studies and heart failure by follow-up echocardiography has rarely been assessed in primary PCI trials. However, the results of the present study support and extend findings from previous studies (19-21) examining the predictors of new-onset heart failure. The results of the present study also demonstrated that LV dysfunction increases the risk for all-cause mortality within one year of follow-up.

Previous studies have identified a number of predictors related to LV dysfunction; however, the data are inconsistent although the majority of studies have identified a larger infarct size at primary PCI. A study by Ezekowitz et al (22) used cardiac magnetic resonance imaging to demonstrate that baseline infarct size was an independent predictor of LV dysfunction 90 days after ST-segment elevation myocardial infarction. A study by Frisch et al (23) identified that periprocedural $\mathrm{LVEF}<30 \%$ was an independent predictor of
LV dysfunction, as was demonstrated in the present study. Therefore, it was hypothesized that the presence of features marking LV dysfunction may confer high risk of experiencing MACEs and by identifying patients who experience persistent myocardial damage and poor clinical outcomes during their index hospitalization, physicians may be able to consider more appropriate use of post-infarction therapies.

The present study demonstrated that the culprit lesion of LAD is also an independent predictor for persistent $\mathrm{LV}$ dysfunction. Anterior myocardial infarctions lead to more pronounced LV dysfunction and more adverse LV remodeling compared with myocardial infarction in other areas (24).

In the present study, troponin I levels were also significantly associated with LV dysfunction. Previous studies have identified an association between troponin elevation and long-term mortality in patients with acute coronary syndrome. A study by Rasoul et al (25) demonstrated that peak cardiac muscle 
Table V. Baseline angiographic characteristics of patients demonstrating a deterioration or improvement of left ventricular function at follow-up echocardiography.

Left ventricular dysfunction

\begin{tabular}{lccc} 
Characteristic & Deterioration $(\mathrm{n}=293)$ & Improvement $(\mathrm{n}=658)$ & P-value \\
\hline Involved vessel, $\%$ & & & 0.703 \\
1-vessel disease & 47.4 & 48.6 & 0.388 \\
2-vessel disease & 31.7 & 28.8 & 0.886 \\
3-vessel disease & 19.1 & 19.4 & 0.422 \\
Left main disease & 1.7 & 3.0 & \\
Lesion target vessel, $\%$ & & & 0.747 \\
Left anterior descending & 53.5 & 54.7 & 0.607 \\
Right coronary & 35.4 & 37.2 & $0.023^{\mathrm{a}}$ \\
Left circumflex & 10.2 & 6.0 & 0.168 \\
Left main & 0.6 & 1.9 & 0.439 \\
ACC/AHA lesion class B2/C, \% & 77.4 & 74.6 & 0.303 \\
Pre-TIMI flow grade 0-1, \% & 68.6 & 65.5 & 0.213 \\
Post-TIMI flow grade 0-1, $\%$ & 2.7 & 1.5 & 0.369 \\
Total stent number & $1.3 \pm 0.6$ & $1.4 \pm 0.7$ & 0.687 \\
Stent number at culprit lesion & $1.0 \pm 0.1$ & $1.0 \pm 0.1$ & 0.455 \\
Stent length, mm & $25.2 \pm 6.2$ & $25.6 \pm 6.3$ & 0.255 \\
Stent diameter, mm & $3.2 \pm 0.3$ & $3.2 \pm 0.4$ &
\end{tabular}

Data are presented as the mean \pm standard deviation unless otherwise specified. ${ }^{a} \mathrm{P}<0.05$. TIMI, thrombolysis in myocardial infarction. ACC/AHA, American College of Cardiology/American Heart Association; TIMI, thrombolysis in myocardial infarction.

troponin $\mathrm{T}(\mathrm{cTnT})$ was negatively correlated with the LVEF measured by myocardial scintigraphy after 3 months and was an independent predictor of heart failure development during a 1-year follow-up. Presentation delay, anterior myocardial infarction location and older age were independent predictors of peak cTnT levels (25). In a study by Hassan et al (26), peak cTnT levels following primary PCI to treat STEMI was also associated with a high incidence of MACEs and heart failure during 1-year clinical follow-up.

The present study had a number of limitations. Firstly, it was not a randomized and controlled study and the non-randomized nature of the registry could result in selection bias. Secondly, subgroup analysis was conducted of all registered patients who had initial and follow-up echocardiography, as well as 1-year clinical outcomes; therefore, many patients who may have qualified for analysis were not included, due to the unavailability of follow-up data, as several patients did not undergo the follow-up echocardiography or were lost to follow-up (2038/4044 patients, 57\%). Thirdly, the initial ejection fraction measured by echocardiography may overestimate the status by sympathetic stimulation, which may result in a difference between initial and follow-up echocardiography. Finally, for unexplained reasons, follow-up echocardiographic studies were not performed on $20 \%$ of total patients, thus selection bias may be present. However, the present study included many more patients than previous studies (19-21) and may be more reliable from that viewpoint. Therefore, further precise, randomized, well-controlled studies are required for more valid conclusions to be drawn.

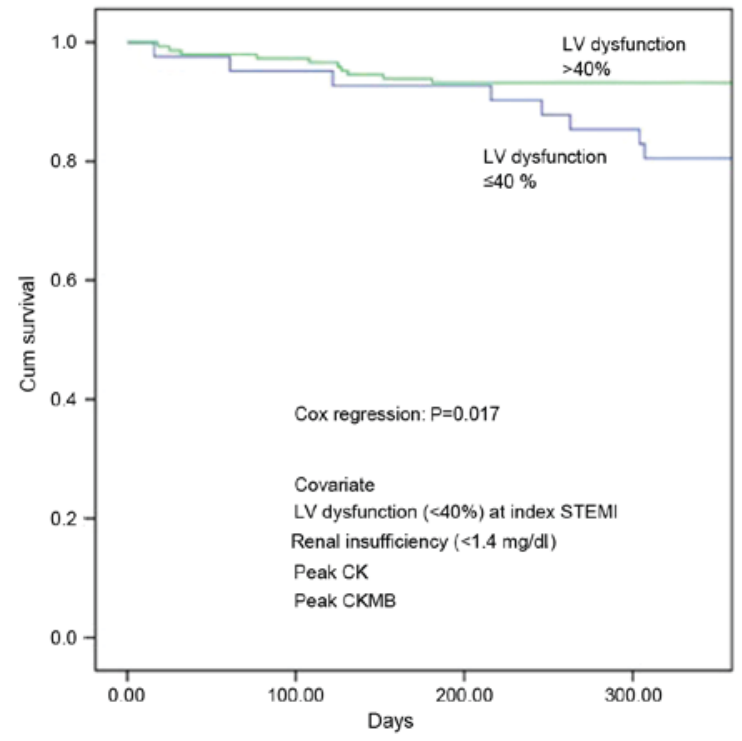

Figure 1. Cumulative survival among patients with LV dysfunction compared to those without LV dysfunction using Cox regression analysis. LV, left ventricular; CK, creatine kinase; CKMB, creatine kinase MB isoenzyme.

In conclusion, persistent LV dysfunction following successful primary PCI is infrequent and is associated with poor clinical outcomes at 1-year clinical follow-up. It may be necessary to evaluate post-infarction patients more meticulously to identify the possibility of persistent LV dysfunction and facilitate more appropriate treatment. 


\section{Acknowledgements}

Not applicable.

\section{Funding}

No funding was received.

\section{Availability of data and materials}

The dataset used and/or analyzed during the current study are available from the corresponding author on reasonable request.

\section{Author's contributions}

DHK was involved in drafting the manuscript. CBP designed the study. DHK, ESJ, HJH and ISS collected and analyzed the data. JMC and CJK interpreted the data and collected the fund for this study and gave final approval of the version to be published. All authors reviewed the initial manuscript and revised it critically for important intellectual content.

\section{Ethics approval and consent to participate}

The present study was approved by the Institutional Review Board at Kyung Hee University Hospital at Gangdong (IRB Approval No. KHNMC 2010-01-065). Written informed consent was obtained from all patients enrolled in the current study.

\section{Consent for publication}

Not applicable.

\section{Competing interests}

The authors declare that they have no competing interests.

\section{References}

1. Levine GN, Bates ER, Blankenship JC, Bailey SR, Bittl JA, Cercek B, Chambers CE, Ellis SG, Guyton RA, Hollenberg SM, et al: 2015 ACC/AHA/SCAI focused update on primary percutaneous coronary intervention for patients with ST-elevation myocardial infarction: An update of the 2011 ACCF/AHA/SCAI guidelines for percutaneous coronary intervention and 2013 ACCF/AHA guideline for the management of ST-elevation myocardial infarction. Circulation 133: 1135-1147, 2016.

2. O'Gara PT, Kushner FG, Ascheim DD, Casey DE Jr, Chung MK, de Lemos JA, Ettinger SM, Fang JC, Fesmire FM, Franklin BA, et al: 2013 ACCF/AHA guideline for the management of ST-elevation myocardial infarction: A report of the American College of Cardiology foundation/American Heart Association task force on practice guideline. Circulation 127: e362-e425, 2013.

3. Mehta RA, Parsons L, Rao SV and Peterson ED; National Registry of Myocardial Infarction (NRMI) Investigators: Association of bleeding and in-hospital mortality in black and white patients with ST-segment elevation myocardial infarction receiving reperfusion. Circulation 125: 1727-1734, 2012.

4. Keely EC, Boura JA and Drines CL: Primary angioplasty versus intravenous thrombolytic therapy for acute myocardial infarction: A quantitative review of 23 randomised trials. Lancet 361: $13-20,2003$.
5. Ibanez B, James S, Agewall S, Antunes MJ, Bucciarelli-Ducci C, Bueno H, Caforio ALP, Crea F, Goudevenos JA, Halvorsen S, et al: 2017 ESC guidelines for the management of acute myocardial infarction in patients presenting with ST-segment elevation: The Task Force for the management of acute myocardial infarction in patients presenting with ST-segment elevation of the European Society of Cardiology (ESC). Eur Heart J 39: 119-177, 2018.

6. Yeh RW, Sidney S, Chandra M, Sorel M, Selby JV and Go AS: Population trends in the incidence and outcomes of acute myocardial infarction. N Eng J Med 362: 2155-2165, 2010.

7. Townsend N, Wilson L, Bhatnagar P, Wickramasinghe K, Rayner M and Nicholas M: Cardiovascular disease in Europe: Epidemiological update 2016. Eur Heart J 37: 3232-3245, 2016.

8. Puymirat E, Simon T, Steg PG, Schiele F, Guéret P, Blanchard D, Khalife K, Goldstein P, Cattan S, Vaur L, et al: Association of changes in clinical characteristics and management with improvement in survival among patients with ST-elevation myocardial infarction. JAMA 308: 998-1006, 2012.

9. Jernberg T, Johanson R, Held C, Svennblad B and Lindback J, Wallentin L; SWEDEHEART/RIKS-HIA: Association between adoption of evidence-based treatment and survival for patients with ST-elevation myocardial infarction. JAMA 305: 1677-1684, 2011.

10. Kelly DJ, Gershlick TG, Witzerbichler B, Gualiumi G, Fahy M, Dangs G, Mehran R and Sone GW: Incidence and predictors of heart failure following percutaneous coronary intervention in ST-segment elevation myocardial infarction: The HORIZON-AMI trial. Am Heart J 162: 663-670, 2011.

11. Spencer FA, Meyer TE, Gore JM and Goldberg RJ: Heterogeneity in the management and outcomes of patients with acute myocardial infarction complicated by heart failure: The National Registry of Myocardial Infarction. Circulation 105: 2605-2610, 2002.

12. Bolognese L, Neskovic AN, Parodi G, Cerisano G, Buoamici P, Santoro GM and Antoniucci D: Left ventricular remodeling after primary coronary angioplasty: Patterns of left ventricular dilation and long-term prognostic implications. Circulation 106: 2351-2357, 2002 .

13. Minicucci MF, Azevedo PS, Polegato BF, Paiva SAR and Zornoff LA: Heart failure after myocardial infarction: Clinical implications and treatment. Clin Cardiol 34: 410-414, 2011.

14. Killip T III and Kimball J: Treatment of myocardial infarction in a coronary care unit. A two year experience with 250 patients. Am J Cardiol 20: 457-464, 1967.

15. Ivanusa $M$ and Milicic D: 40 years since Killip clinical classification. Int J Cardiol 134: 420-421, 2009.

16. Steg PG, Dabbous OH, Feldman LJ, Cohen-Solal A, Aumont MC, Lopez-Sendon J, Budaj A, Goldberg RJ, Klein W, Anderson FA Jr, et al: Determinants and prognostic impact of heart failure complicating acute coronary syndromes: Observations from the Global registry of acute coronary events (GRACE). Circulation 109: 494-499, 2004.

17. Wu AH, Parsons L, Every NR and Bates ER; Second National Registry of Myocardial Infarction: Hospital outcomes in patients presenting with congestive heart failure complicating acute myocardial infarction: A report from the Second National registry of myocardial infarction (NRMA-2). J Am Coll Cardiol 40: 1389-1394, 2002.

18. Mandelzweig L, Battler A, Boyko V, Bueno H, Danchin N, Filippatos G, Gitt A, Hasdai D, Hasin Y, Marrugat J, et al: The second Euro Heart Survey on acute coronary syndromes: Characteristics, treatment, and outcome of patients with ACS in Europe and the Mediterranean basin in 2004. Eur Heart J 27: 2285-2293, 2006.

19. Parodi G, Memisha G, Carrabba N, Signorini U, Migliorini A, Cerisano G and Antoniucci D: Prevalence, predictors, time course and long-term clinical implications of left ventricular functional recovery after mechanical reperfusion for acute myocardial infarction. Am J Cardiol 100: 1718-1722, 2007.

20. Parodi G and Antoniucci D: Left ventricular remodeling after primary percutaneous coronary intervention. Am Heart J 160 (6 Suppl): S11-S15, 2010.

21. Bolognese L, Neskovic N, Parodi G, Cerisano G, Buonamici P, Santoro GM and Antoniucci D: Left ventricular remodelling after primary coronary angioplasty: Patterns of left ventricular dilation and long-term prognostic implications. Circulation 106: 2351-2357, 2002 . 
22. Ezekowitz JA, Armastrong PW, Granger CB, Theroux P, Stebbins A, Kim RJ and Patel MR: Predicting chronic left ventricular dysfunction 90 days after ST-segment elevation myocardial infarction: An assessment of pexelizumab in acute myocardial infarction (APEX-AMI) substudy. Am Heart J 160: 272-278, 2010

23. Frisch DR, Giedrimas E, Mohanavelu S, Shui A, Ho KK, Gibson CM, Josephson ME and Zemitbaum PJ: Predicting irreversible left ventricular dysfunction after acute myocardial infarction. Am J Cardiol 103: 1206-1209, 2009.

24. Masci PG, Ganame J, Francone M, Desmet W, Lorenzoni V, Iacucci I, Barison A, Carbone I, Lombardi M, Agati L, et al: Relationship between location and size of myocardial infarction and their reciprocal influences on post-infarction left ventricular remodeling. Eur Heart J 32: 1640-1648, 2011

25. Rasoul S, Nienhuis MB, Ottervanger JP, Slingerland RJ, de Boer MJ, Dambrink JH, Ernst NM, Hoorntje JC, Gosselink AT, Suryapranta $\mathrm{H}$, et al: Predictors of elevated cardiac troponin-T on admision ST-segment elevation myocardial infarction. Ann Clin Biochem 43: 281-286, 2006.
26. Hassan AK, Bergheanu SC, Hasan-Ali H, Liem SS, van der Laarse A, Wolterbeek R, Atsma DE, Schalij MJ and Jukema JW: Usefulness of peak troponin-T to predict infarct size and long-term outcome in patients with first acute myocardial infarction after primary percutaneous coronary intervention. Am J Cardiol 103: 779-784, 2009.

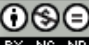

This work is licensed under a Creative Commons Attribution-NonCommercial-NoDerivatives 4.0 International (CC BY-NC-ND 4.0) License. 